https://doi.org/10.5194/hess-2019-622

Preprint. Discussion started: 3 January 2020

(C) Author(s) 2020. CC BY 4.0 License.

\title{
1 A reduced-order model for dual state-parameter geostatistical inversion
}

2 Yu-Li Wang ${ }^{1}$, Tian-Chyi Jim Yeh ${ }^{1}$, Jui-Pin Tsai ${ }^{2, *}$

3

4 1. Department of Hydrology and Atmospheric Sciences, University of Arizona,

5 Arizona, United States.

6 2. Department of Bioenvironmental Systems Engineering, National Taiwan

7 University, Taiwan.

8

$9 *$ Corresponding Author

10 Assistant Professor Jui-Pin Tsai, jptsai@ ntu.edu.tw

11

12 


\section{Abstract}

To properly account the subsurface heterogeneity, geostatistical inverse models usually permit enormous amount of spatial correlated parameters to interpret the collected states. Several reduced-order techniques for the brick domain are investigated to leverage the memory burden of parameter covariance. Their capability to irregular domain is limited. Furthermore, due to the over fitting of states, the estimated parameters usually diverge to unreasonable values. Although some propriate tolerances can be used to eliminate this problem, they are presumed and heavily rely on the personal judgement. To address these two issues, we present a model reduction technique to the irregular domain by singular value decomposition (SVD). Afterward, the state errors and parameters are sequentially updated to leverage the over fitting. The computational advantages of the proposed reduced-order dual state-parameter inverse algorithm are demonstrated through two numerical experiments and one case study in a catchment scale field site. The investigations suggest that the stability of convergence dramatically improves. The estimated parameter values stabilize to reasonable order of magnitude. In addition, the memory requirement significantly reduces while the resolution of estimate preserves. The proposed method benefits multi-discipline scientific problems, especially useful and convenient for assimilating different types of measurements. 


\section{Introduction}

Groundwater is one of the necessary resources in many regions where the amount of rainfall and the capacity of reservoir is limited. To provide enough fresh water for the current and future uses in these areas, proper water resources management and contaminated site remediation strategies are required, which relies on the understanding of the site-specific spatial distribution of hydrological parameters (e.g., hydraulic conductivity and specific storage) in the prefer scale.

Many covariance based geostatistical approaches have been widely employed for aquifer characterization. Several previous studies suggested that the geostatistical inversion is superior than many other subsurface inverse modeling because it estimates the uncertainty and has ability to assimilate different type of observed data sequentially (Vesselinov et al., 2001). However, as pointed out by Illman et al. (2015), when the number of observations and unknown parameters are huge, the primary drawbacks of geostatistical inversion are the computational and memory burdens.

Several ensemble approaches have been proposed to handle the memory and large covariance matrices. For instance, Particle Filter or Sequential Monte Carlo method (SMC, Field et al., 2016; Zhang et al., 2017), iterative Ensemble Kalman Filter (EnKF, Schöniger et al., 2012; Ait-El-Fquih et al., 2016), iterative Ensemble Smoother (ES, Zhang et al., 2018), Extended Kalman Filter (EKF, Yeh and Huang 2005; Leng and Yeh, 2003), and many other related methods construct the covariance between the parameter and state variable from a set of ensemble member. Since a bunch of realizations (usually several hundreds or thousands) are required to infer the population covariance from the sample covariance, the algorithm may not be computational affordable when the simulation time of single forward modeling is time consuming. 
61 large set of ensemble realizations. They construct the parameter covariance by some

62 prior knowledge of unknown parameter field (e.g., covariance function, variance, and

63 correlation length). Afterward, the covariance between the parameter and state

64 variable is estimated through the sensitivity of state variable with respect to parameter.

65 This approach requires significant amount of memory resource when the number of

66 unknown parameter and state variable are huge. Furthermore, evaluating the

67 sensitivity efficiently may be a difficult task for some scientific problems. As a result,

68 considerable efforts are devoted to improving the capability of the algorithm. For

69 instance, Sun and Yeh (1990) employed the adjoint approach to evaluate the

70 sensitivity. It reduces the cost of running forward model from the order of number of

71 unknown parameters to the number of state measurements. Saibaba and Kitanidis

72 (2012) incorporates the hierarchical matrices technique with a matrix-free Krylov

73 subspace approach to improve the computational efficiency. Liu et al. (2014) avoids

74 the direct solution of sensitivity matrix by the Krylov subspace method. Li et al. (2015)

75 and Zha et al. (2018) project the covariance matrix on the orthonormal basis and

76 evaluate the cross product of sensitivity and squared root covariance directly using

77 finite differencing approach. This method eliminates the sensitivity evaluation and

78 reduces the computational cost of running forward model to the order of number of

79 leading modes. Li et al. (2014) take the advantage of hierarchical nature of matrices to

80 accelerate the computation of dense matrix vector products and rewrite the Kalman

81 filtering equations into a computational efficient manner. Ghorbanidehno et al. (2015)

82 extend their approach to the general case of non-linear dynamic systems. Similarly,

83 Lin et al. (2016) reduces the computational complexity by projecting the parameters

84 to different hierarchies of Krylov subspace. Pagh (2013) use fast Fourier transform to

85 speed up the computation of covariance matrix multiplication. In addition, many 
86 approaches reduce the computational cost and memory requirement. For example,

87 Nowak and Litvinenko (2013) combine low rank approximations to the covariance

88 matrices with fast Fourier transform; Kitanidis (2015) decomposes the covariance matrix by some orthonormal basis and shows that the choice of basis can be tailored to the problem of interest to improve estimation accuracy; Li et al. (2015) use discrete cosine transform to compress the data covariance matrix of a 1-D state variable series; Zha et al. (2018) use Karhunen-Loeve Expansion to compress the parameter covariance matrix of a 3-D parameter field. Other useful reduced order models are Galerkin projection (Liu et al., 2013), principal component (Kitanidis and Lee, 2014), randomized algorithm (Lin et al., 2017), Whittaker-Shannon interpolation (Horning et al., 2019), and Kronecker product decomposition (Zunino and Mosegaard, 2019).

In addition to reformulate the covariance matrix, the temporal moments eliminate the temporal derivative term in the governing equation. Thus, it is another potential method to reduce the data size and computational cost (Cirpka and Kitanidis, 2000; Nowak and Cirpka, 2006; Yin and Illman, 2009).

There are several limitations exist in the previous geostatistical inverse algorithms. The first issue is over calibration or over fitting. During the inverse process, the calibration terminates when the difference between the observed and simulated states reduces to the value smaller than the given tolerance, an arbitrary value based on user's personal judgement. In practical, the tolerance is determined by the expected numeric and measurement errors. Since its true order of magnitude is unknow, the estimated parameter field sometimes diverges if the tolerance is underestimated. To be specific, the estimated parameters will first converge to the best values accompanied with the successive assimilation of the information about the subsurface heterogeneity embedded in the observed state variables. The values of parameter then diverge to the unreasonable huge or small values to compensate the 
112 numeric and measurement errors. This instability is not user friendly because the

113 reasonable (i.e., converged) estimate needs to be selected manually. Furthermore,

114 when different types of measurement (e.g., water level, flux, temperature, gravity, etc.)

115 are available, it is suggested that assimilate these data sequentially is a more robust

116 approach than the simultaneous assimilation (Tsai et al., 2017). Accordingly, the

117 manually determination of convergence prohibits the automatic sequential

118 assimilation.

119 Second, when dealing with a 2-D or 3-D parameter or state variable fields, a 120 specific matrix structures are required to efficiently decompose the unconditional 121 covariance matrix to the orthonormal basis. For instance, a regular grid spacing is 122 required to efficiently perform the fast Fourier transform (Nowak and Litvinenko, 123 2013) and discrete cosine transform (Li et al., 2015). Similarly, Karhunen-Loeve 124 Expansion (Zha et al., 2018) requires a brick or rectangle shape domain and grid. This 125 requirement comes from the derivation of analytic eigenvalue and eigenvector of a

126 separable exponential function.

To overcome these two existing limitations, we first introduce an additional step to estimate the error of state variables based on the error covariance matrices. Next, we derive a reduced order model using singular value decomposition. Afterward, we present a matrix manipulation method to eliminate the requirement of brick or rectangle domain during constructing the eigenvalue and eigenvector of unconditional covariance matrix.

This paper is arranged as follows. We first revisit the SLE that forms the geostatistical inversion approach (section 2.2). Thereafter, the algorithm is reformulated by collaborating with the data error, reduced order approach (i.e. singular value decomposition, SVD), and irregular domain (section 2.3). Furthermore, a perturbation method is proposed to improve the efficiency of covariance evaluation 
138 process (section 2.4). In section 3, we test the proposed dual state-parameter

139 estimation algorithm with two synthetic examples and a tomographic survey at the

140 field site to demonstrate the superiority of the proposed method. Lastly, summary and

141 conclusions are presented.

142

143

\subsection{Groundwater Flow Model.}

144 The 2-D groundwater flow in heterogeneous confined aquifer can be described 145 as

$$
\nabla \cdot[T(\mathbf{x}) \cdot \nabla h(\mathbf{x}, t)]=S(\mathbf{x}) \frac{\partial h(\mathbf{x}, t)}{\partial t}
$$

147

148

149

150

151

152

and

\subsection{Reduced order successive linear estimator}

The singular value decomposition (SVD) is employed to reduce the order of the parameter covariance, leading to less memory requirement and more computational efficiency inverse exercise. Afterward, the data error is considered to improve the stability of convergence.

\section{(1) Hard Data}

When the hard data are available, kringing is used to estimate the conditional parameter field and the corresponding conditional covariance matrix from the measured parameters. It is expressed as

$$
\hat{\mathbf{f}}^{(1)}=\hat{\mathbf{f}}^{(0)}+\boldsymbol{\varepsilon}_{f f}^{(0)} \mathbf{C} \mathbf{R}_{f^{*} f^{*}}^{-1}\left[\mathbf{f}^{*}-\hat{\mathbf{f}}^{(0)}\right]
$$

$$
\boldsymbol{\varepsilon}_{f f}^{(1)}=\boldsymbol{\varepsilon}_{f f}^{(0)}-\boldsymbol{\varepsilon}_{f f}^{(0)} \mathbf{C R}_{f^{*} f^{*}}^{-1} \mathbf{C}^{T} \boldsymbol{\varepsilon}_{f f}^{(0)}
$$


162 in which $\mathbf{f}^{*}\left(n_{m} \times 1\right)$ is the measured parameters, $\hat{\mathbf{f}}^{(0)}\left(n_{f} \times 1\right)$ is the unconditional 163 parameter field, $\hat{\mathbf{f}}^{(1)}\left(n_{f} \times 1\right)$ is the conditional parameter field. $n_{f}$ represents the 164 number of unknown parameters and $n_{m}$ represents the number of measured parameters.

$165 \boldsymbol{\varepsilon}_{f f}^{(0)} \mathbf{C}$ and $\mathbf{C}^{T} \boldsymbol{\varepsilon}_{f f}^{(0)}\left(n_{m} \times n_{f}\right)$ are the unconditional parameter covariance matrices 166 depicting the spatial correlation between the measured parameters and all parameters. $167 \boldsymbol{\varepsilon}_{f f}^{(0)}\left(n_{f} \times n_{f}\right)$ is the unconditional parameter covariance matrix depicting the spatial 168 correlation between all parameters $(\hat{\mathbf{f}}) . \mathbf{C}\left(n_{f} \times n_{m}\right)$ is a matrix eliminating the column 169 in $\boldsymbol{\varepsilon}_{f f}^{(0)}$ when the corresponding measured parameter is absent. $\boldsymbol{\varepsilon}_{f f}^{(1)}\left(n_{f} \times n_{f}\right)$ is the 170 conditional covariance marix of all parameters. The diagonal term of the matrix (i.e., 171 residual variance) represents the remaining uncertainty of the estimated parameter 172 after the information (measurements) is included. A small residual variance indicates 173 the spatial trend of estimated parameter is close to the true, while a large value 174 indicates the estimate is close to the initial guessed value (i.e. heterogeneity is not 175 resolved). $\mathbf{R}_{f^{*} f^{*}}\left(n_{m} \times n_{m}\right)$ is the covariance matrix depicting the correlation between 176 measured parameters. Notice that Cholesky and QR decompositions are utilized to 177 solve the matrix multiplication of inverse $\mathbf{R}_{f^{*} f^{*}}$ when it is and is not a positive 178 definite matrix.

179 Since $n_{f}$ is usually huge, storage demand of $\boldsymbol{\varepsilon}_{f f}^{(0)}$ and $\boldsymbol{\varepsilon}_{f f}^{(1)}$ may not be always 180 affordable. Thus, singular value decomposition (SVD) is utilized to relieve this 181 memory burden by keeping the leading eigenvalues and eigenvectors. The SVD of $182 \boldsymbol{\varepsilon}_{f f}$ is expressed as

$$
\boldsymbol{\varepsilon}_{f f}=\mathbf{g} \boldsymbol{\lambda} \mathbf{g}^{T}
$$

184 where $\lambda\left(n_{s v d} \times n_{s v d}\right)$ is eigenvalues, $\mathbf{g}\left(n_{f} \times n_{s v d}\right)$ is eigenvectors, and $n_{s v d}$ is number 
185 of leading eigenvalues. Substitute eq. (5) into eqs. (3) and (4), we have

$$
\hat{\mathbf{f}}^{(1)}=\hat{\mathbf{f}}^{(0)}+\mathbf{g}^{(0)} \boldsymbol{\lambda}^{(0)} \mathbf{g}^{(0) T} \mathbf{C} \mathbf{R}_{f f}^{-1}\left[\mathbf{f}^{*}-\hat{\mathbf{f}}^{(0)}\right]
$$

187 and

$$
\mathbf{g}^{(1)} \boldsymbol{\lambda}^{(1)} \mathbf{g}^{(1) T}=\mathbf{g}^{(0)} \boldsymbol{\lambda}^{(0)} \mathbf{g}^{(0) T}-\mathbf{g}^{(0)} \boldsymbol{\lambda}^{(0)} \mathbf{g}^{(0) T} \mathbf{C} \mathbf{R}_{f f}^{-1} \mathbf{C}^{T} \mathbf{g}^{(0)} \boldsymbol{\lambda}^{(0)} \mathbf{g}^{(0) T}
$$

189 Since $\mathbf{g}^{(1)}$ is always a function of $\mathbf{g}^{(0)}$, it can be expressed as

$$
\mathbf{g}^{(1)}=\mathbf{g}^{(0)} \mathbf{u}^{(0)}
$$

191 where is $\mathbf{u}^{(0)}\left(n_{s v d} \times n_{s v d}\right)$ is the matrix transferring the information of spatial correlation of parameters to the next iteration. Accordingly, eq. (7) can reduce to

$$
\mathbf{u}^{(0)} \boldsymbol{\lambda}^{(1)} \mathbf{u}^{(0) T}=\sqrt{\boldsymbol{\lambda}^{(0)}}\left(\mathbf{I}-\sqrt{\lambda^{(0)}} \mathbf{g}^{(0) T} \mathbf{C R}_{f^{*} f^{*}}^{-1} \mathbf{C}^{T} \mathbf{g}^{(0)} \sqrt{\boldsymbol{\lambda}^{(0)}}\right) \sqrt{\boldsymbol{\lambda}^{(0)}}
$$

194 in which I is an identity matrix. By decomposing eq. (9) with SVD, we obtain the

195 updated eigenvalue $\lambda^{(1)}$ and $\mathbf{u}^{(0)}$. The updated eigenvector $\mathbf{g}^{(1)}$ can be evaluated 196 by eq. (8).

After considering the hard data, the inherently presented data errors (e.g., measurement, numeric, round-off, truncation errors, etc.) are included prior to the parameter estimation. The estimated data error and the corresponding covariance matrix are expressed as

$$
\hat{\mathbf{e}}^{(r+1)}=\hat{\mathbf{e}}^{(r)}+\boldsymbol{\varepsilon}_{h h}^{(r)}\left[\mathbf{J}_{f h}^{(r) T} \boldsymbol{\varepsilon}_{f f}^{(r)} \mathbf{J}_{f h}^{(r)}+\boldsymbol{\varepsilon}_{h h}^{(r)}\right]^{-1}\left[\mathbf{h}^{(r)}-\left(\mathbf{h}^{*}+\hat{\mathbf{e}}^{(r)}\right)\right]
$$

and

$$
\boldsymbol{\varepsilon}_{h h}^{(r+1)}=\boldsymbol{\varepsilon}_{h h}^{(r)}-\boldsymbol{\varepsilon}_{h h}^{(r)}\left[\mathbf{J}_{f h}^{(r) T} \boldsymbol{\varepsilon}_{f f}^{(r)} \mathbf{J}_{f h}^{(r)}+\boldsymbol{\varepsilon}_{h h}^{(r)}\right]^{-1} \boldsymbol{\varepsilon}_{h h}^{(r)}
$$

205 in which $\mathbf{h}^{*}\left(n_{d} \times 1\right)$ is the observed head and $\mathbf{h}^{(r)}\left(n_{d} \times 1\right)$ is the simulated head 206 based on the estimated parameters from the $r^{\text {th }}$ iteration. $n_{d}$ represents the number of 207 measured state variables. The superscript $r$ is the iteration index starting from one. 
$208 \boldsymbol{\varepsilon}_{h h}^{(1)}\left(n_{d} \times n_{d}\right)$ is the unconditional covariance matrix of the observed head. The

209 diagonal terms represent the uncertainty of the measurement and the off diagonal

210 terms represent the correlation between errors. $\boldsymbol{\varepsilon}_{h h}^{(r)}$ and $\boldsymbol{\varepsilon}_{h h}^{(r+1)}\left(n_{d} \times n_{d}\right)$ are the

211 conditional covariance matrices. $\hat{\mathbf{e}}^{(1)}\left(n_{d} \times 1\right)$ is the initial data error. $\hat{\mathbf{e}}^{(r)}$ and $\hat{\mathbf{e}}^{(r+1)}$

$212\left(n_{d} \times 1\right)$ are the estimated data error. $\mathbf{J}_{f h}^{(r)}\left(n_{f} \times n_{d}\right)$ is the sensitivity of observed head

213 with respect to the estimated parameters during the $r^{\text {th }}$ iteration.

214 The weight (i.e., $\mathbf{W}^{(r)}=\boldsymbol{\varepsilon}_{h h}^{(r)}\left[\mathbf{J}_{f h}^{(r) T} \boldsymbol{\varepsilon}_{f f}^{(r)} \mathbf{J}_{f h}^{(r)}+\boldsymbol{\varepsilon}_{h h}^{(r)}\right]^{-1}$ ) is a combination of observed

215 head covariance matrix $\left(\boldsymbol{\varepsilon}_{h h}^{(r)}\right)$ and simulated head covariance matrix

$216\left(\mathbf{R}_{h h}^{(r)}=\mathbf{J}_{f h}^{(r) T} \boldsymbol{\varepsilon}_{f f}^{(r)} \mathbf{J}_{f h}^{(r)}\right)$. It represents the ratio of data error $\left(\boldsymbol{\varepsilon}_{h h}^{(r)}\right.$, including numeric and

217 measurement errors) to the total error $\left(\mathbf{R}_{h h}^{(r)}+\boldsymbol{\varepsilon}_{h h}^{(r)}\right.$, including model structure,

218 parameter, numeric, and measurement errors). When the model is poorly calibrated,

219 the simulated head based on the current model structure and parameter values is much

220 uncertain than that of observed head (i.e. $\mathbf{R}_{h h}^{(r)}>>\boldsymbol{\varepsilon}_{h h}^{(r)}$ ). Thus, the weight ( $\mathbf{W}^{(r)}$ ) is

221 small and the algorithm trusts the observation $\left(\mathbf{h}^{*}\right)$ more than the prediction $\left(\mathbf{h}^{(r)}\right)$.

222 After assimilating the subsurface characteristic casted in the observation, the

223 uncertainty of simulated head $\left(\mathbf{R}_{h h}^{(r)}\right)$ reduces and the algorithm trusts the observation

$224\left(\mathbf{h}^{*}\right)$ less than the prediction $\left(\mathbf{h}^{(r)}\right)$. Therefore, dismiss between $\mathbf{h}^{*}$ and $\mathbf{h}^{(r)}$ are

225 reflected into $\hat{\mathbf{e}}^{(r)}$. This data error calibration step is similar to the Kalman filter, but

226 instead of using the observation from previous time step only, we consider all of the

227 available observation simultaneously.

228 Again, substitute eigenvalue $\lambda$ and eigenvector $\mathbf{g}$ of $\boldsymbol{\varepsilon}_{f f}^{(r)}$ expressed in eq.

229 (5), the reduced order formulations of eqs. (10) and (11) are 
231 and

$$
\boldsymbol{\varepsilon}_{h h}^{(r+1)}=\boldsymbol{\varepsilon}_{h h}^{(r)}-\boldsymbol{\varepsilon}_{h h}^{(r)}\left[\mathbf{H}_{f h}^{(r)} \mathbf{H}_{f h}^{(r) T}+\boldsymbol{\varepsilon}_{h h}^{(r)}\right]^{-1} \boldsymbol{\varepsilon}_{h h}^{(r)}
$$

233 where $\mathbf{H}_{f h}^{(r)}\left(n_{d} \times n_{s v d}\right)$ is

$$
\mathbf{H}_{f h}^{(r)}=\mathbf{J}_{f h}^{(r) T} \mathbf{g}^{(r)} \sqrt{\lambda^{(r)}}
$$

235 Notice that if the number of state $\left(n_{d}\right)$ is huge, SVD can potentially be used to 236 decompose $\boldsymbol{\varepsilon}_{h h}^{(r)}$ (eq. 5) and reduce the storage requirement.

238 After estimating the data error, the measured state variables and data errors are 239 substituted into successive linear estimator (SLE) (Yeh et al., 1996) to estimate the

240 conditional parameter fields and the corresponding residual covariance matrix:

$$
\hat{\mathbf{f}}^{(r+1)}=\hat{\mathbf{f}}^{(r)}+\boldsymbol{\varepsilon}_{f f}^{(r)} \mathbf{J}_{f h}^{(r)}\left[\mathbf{J}_{f h}^{(r) T} \boldsymbol{\varepsilon}_{f f}^{(r)} \mathbf{J}_{f h}^{(r)}+\boldsymbol{\varepsilon}_{h h}^{(r+1)}\right]^{-1}\left[\left(\mathbf{h}^{*}+\hat{\mathbf{e}}^{(r+1)}\right)-\mathbf{h}^{(r)}\right]
$$

242

and

$$
\boldsymbol{\varepsilon}_{f f}^{(r+1)}=\boldsymbol{\varepsilon}_{f f}^{(r)}-\boldsymbol{\varepsilon}_{f f}^{(r)} \mathbf{J}_{f h}^{(r)}\left[\mathbf{J}_{f h}^{(r) T} \boldsymbol{\varepsilon}_{f f}^{(r)} \mathbf{J}_{f h}^{(r)}+\boldsymbol{\varepsilon}_{h h}^{(r+1)}\right]^{-1} \mathbf{J}_{f h}^{(r) T} \boldsymbol{\varepsilon}_{f f}^{(r)}
$$

(15) and (16). That is,

246

$$
\hat{\mathbf{f}}^{(r+1)}=\hat{\mathbf{f}}^{(r)}+\mathbf{g}^{(r)} \sqrt{\lambda^{(r)}} \mathbf{H}_{f h}^{(r) T}\left[\mathbf{H}_{f h}^{(r)} \mathbf{H}_{f h}^{(r) T}+\boldsymbol{\varepsilon}_{h h}^{(r+1)}\right]^{-1}\left[\left(\mathbf{h}^{*}+\hat{\mathbf{e}}^{(r+1)}\right)-\mathbf{h}^{(r)}\right]
$$

247 and

248

$$
\mathbf{g}^{(r+1)} \boldsymbol{\lambda}^{(r+1)} \mathbf{g}^{(r+1) T}=\mathbf{g}^{(r)} \boldsymbol{\lambda}^{(r)} \mathbf{g}^{(r) T}-\mathbf{g}^{(r)} \sqrt{\boldsymbol{\lambda}^{(r)}} \mathbf{H}_{f h}^{(r) T}\left[\mathbf{H}_{f h}^{(r)} \mathbf{H}_{f h}^{(r) T}+\boldsymbol{\varepsilon}_{h h}^{(r+1)}\right]^{-1} \mathbf{H}_{f h}^{(r)} \sqrt{\boldsymbol{\lambda}^{(r)}} \mathbf{g}^{(r) T}
$$

249 Using eq. (8), eq. (18) further reduces to

$$
\mathbf{u}^{(r)} \boldsymbol{\lambda}^{(r+1)} \mathbf{u}^{(r) T}=\sqrt{\boldsymbol{\lambda}^{(r)}}\left(\mathbf{I}-\mathbf{H}_{f h}^{(r) T}\left[\mathbf{H}_{f h}^{(r)} \mathbf{H}_{f h}^{(r) T}+\boldsymbol{\varepsilon}_{h h}^{(r+1)}\right]^{-1} \mathbf{H}_{f h}^{(r)}\right) \sqrt{\lambda^{(r)}}
$$

251 By decomposing eq. (19) with SVD, we can evaluate the updated eigenvalue $\lambda^{(r+1)}$ 
252 and $\mathbf{u}^{(r)}$. The updated eigenvector $\mathbf{g}^{(r+1)}$ then can be calculated by eq. (8).

253

\section{(4) Convergence Criterion}

The estimated field is considered as the converge one when the spatial variance of the estimated parameter duing several iterations are steady. The tolarence using mean squared error between the observed and simulated states is no longer necessary.

\subsection{Required Inputs}

To initiate the algorithm, the initial guess of parameter field $\hat{\mathbf{f}}^{(0)}$ and data error $\hat{\mathbf{e}}^{(0)}$, as well as the unconditional covariance matrix of the parameters $\boldsymbol{\varepsilon}_{f f}^{(0)}$ and observed data $\boldsymbol{\varepsilon}_{h h}^{(0)}$ are required. The details are explained as followings:

Parameter Field: The initial parameter field $\hat{\mathbf{f}}^{(0)}$ can be any reasonable values based on the prior knowledge.

Parameter Covariance: We assume the unconditional parameter covariance matrix is defined by an exponential covariance function

$$
\boldsymbol{\varepsilon}_{f f}^{(0)}=V a r \cdot \exp \left(\frac{-\left|\mathbf{d}_{\mathbf{x}}\right|}{\lambda_{x}}+\frac{-\left|\mathbf{d}_{\mathbf{y}}\right|}{\lambda_{y}}\right)
$$

where $\operatorname{Var}$ represents the unconditional spatial variance of the parameter; $\mathbf{d}_{\mathbf{x}}\left(n_{f} \times 1\right)$ and $\mathbf{d}_{\mathbf{y}}\left(n_{f} \times 1\right)$ are the distance between two parameters in $x$ and $y$ directions; $\lambda_{x}$ and $\lambda_{y}$ are the correlation lengths $(\mathrm{m})$ in $x$ and $y$ directions.

The reduced order algorithm requires the evaluation of unconditional parameter covariance matrix $\boldsymbol{\varepsilon}_{f f}^{(0)}$ in terms of eigenvalue $\lambda^{(0)}$ and eigenvector $\mathbf{g}^{(0)}$. In the real-world problem, the number of parameters $n_{f}$ is usually in the order of $10^{3}$ to $10^{5}$, and the computational cost of conducting full SVD is $n_{f}^{3}\left(O\left(n_{f}^{3}\right)\right)$. Alternately, 
274 truncated SVD with the complexity in $O\left(n_{f t}^{2} n_{f}\right)$ can be used to approximate the

275 original eigenvalue and eigenvector. $n_{f t}$ is the number of randomly chose column in $276 \boldsymbol{\varepsilon}_{f f}^{(0)}$.

277 In addition to the numeric approach, the analytical solution of eigenvalues $\lambda_{n}$

278 and eigenvectors $\mathbf{g}_{n}$ with brick grid and domain (Ghanem and Spanos, 2003; Zhang

279 and $\mathrm{Lu}, 2004)$ is also available. In 2-D domain, they are analytically express as

$$
\lambda_{n}=\operatorname{Var} \frac{2 \lambda_{x}}{\lambda_{x}^{2} w_{n, x}^{2}+1} \frac{2 \lambda_{y}}{\lambda_{y}^{2} w_{n, y}^{2}+1}(21)
$$

$$
\mathbf{g}_{n}=\frac{\lambda_{x} w_{n, x} \cos \left(w_{n, x} x\right)+\sin \left(w_{n, x} x\right)}{\sqrt{\frac{\left(\lambda_{x}^{2} w_{n, x}^{2}+1\right) L_{x}}{2}+\lambda_{x}}} \frac{\lambda_{y} w_{n, y} \cos \left(w_{n, y} y\right)+\sin \left(w_{n, y} y\right)}{\sqrt{\frac{\left(\lambda_{y}^{2} w_{n, y}^{2}+1\right) L_{y}}{2}+\lambda_{y}}}
$$

282

where $w_{n, x}$ and $w_{n, y}$ are the positive roots of the characteristic equations

$$
\left(\lambda_{x}^{2} w_{n, x}^{2}-1\right) \sin \left(w_{n, x} L_{x}\right)=2 \lambda_{x} w_{n, x} \cos \left(w_{n, x} L_{x}\right)
$$

and

$$
\left(\lambda_{y}^{2} w_{n, y}^{2}-1\right) \sin \left(w_{n, y} L_{y}\right)=2 \lambda_{y} w_{n, y} \cos \left(w_{n, y} L_{y}\right)
$$

where $L_{x}$ and $L_{y}$ are the width of model domain in $x$ and $y$ directions.

Notice that if the model domain is irregular (i.e., not a line, squared, or brick shape), one can first construct the eigenvalue and eigenvector for a regular domain whose size is greater than the irregular one. Afterward, the eigenvector of the irregular domain can be evaluated by

$91 \quad \mathbf{g}_{\text {irreg }}=\mathbf{C}_{2} \mathbf{g}_{\text {reg }}$ (25) in which $\mathbf{C}_{2}\left(n_{f, \text { irreg }} \times n_{f, r e g}\right)$ is a matrix to eliminate the rows of $\mathbf{g}_{\text {reg }}$ if the corresponding grids are outside the model domain; $n_{f, \text { reg }}$ is the number of parameter of 
294 the regular line, squared, or brick domain; $n_{f, i r r e g}$ is the number of parameter of the

295 irregular domain. $\mathbf{g}_{\text {reg }}$ and $\mathbf{g}_{\text {irreg }}$ are the eigenvectors of regular and irregular 296 domains.

297 Data Error: The initial data error $\hat{\mathbf{e}}^{(0)}$ can set as zero.

298 Data Covariance: The unconditional covariance matrix of the observed data $299 \boldsymbol{\varepsilon}_{h h}^{(0)}$ is a diagonal matrix if the data error are mutually independent. Otherwise, a 300 covariance function (e.g., eq. (20)) can be utilized to describe the unconditional 301 correlation.

302

303

304

305

306

307

$$
G\left(\hat{\mathbf{f}}^{(r)}+\mathbf{g}^{(r)} \delta\right)=G\left(\hat{\mathbf{f}}^{(r)}\right)+G^{\prime}\left(\hat{\mathbf{f}}^{(r)}\right) \mathbf{g}^{(r)} \delta+G^{\prime \prime}\left(\hat{\mathbf{f}}^{(r)}\right) \frac{\left(\mathbf{g}^{(r)} \delta\right)^{2}}{2}+G^{\prime \prime \prime}\left(\hat{\mathbf{f}}^{(r)}\right) \frac{\left(\mathbf{g}^{(r)} \delta\right)^{3}}{3 !}+\ldots
$$

$316 \delta$ is an arbitrary value controlling the accuracy of approximation. Manipulating eq. 
317 (26) yields

$318 G^{\prime}\left(\hat{\mathbf{f}}^{(r)}\right) \mathbf{g}^{(r)}=\mathbf{J}_{f h}^{(r) T} \mathbf{g}^{(r)}=\frac{G\left(\hat{\mathbf{f}}^{(r)}+\mathbf{g}^{(r)} \delta\right)-G\left(\hat{\mathbf{f}}^{(r)}\right)}{\delta}-G^{\prime \prime}\left(\hat{\mathbf{f}}^{(r)}\right) \frac{\left(\mathbf{g}^{(r)}\right)^{2} \delta}{2}-G^{\prime \prime \prime}\left(\hat{\mathbf{f}}^{(r)}\right) \frac{\left(\mathbf{g}^{(r)}\right)^{3} \delta^{2}}{3 !}-\ldots$

$319 \quad(27)$

320 Multiplying both sides with $\sqrt{\lambda^{(r)}}$, eq. (27) becomes

$321 \quad \mathbf{H}_{f h}^{(r)}=\left[\frac{G\left(\hat{\mathbf{f}}^{(r)}+\mathbf{g}^{(r)} \delta\right)-G\left(\hat{\mathbf{f}}^{(r)}\right)}{\delta}-G^{\prime \prime}\left(\hat{\mathbf{f}}^{(r)}\right) \frac{\left(\mathbf{g}^{(r)}\right)^{2} \delta}{2}-G^{\prime \prime \prime}\left(\hat{\mathbf{f}}^{(r)}\right) \frac{\left(\mathbf{g}^{(r)}\right)^{3} \delta^{2}}{3 !}-\ldots\right] \sqrt{\lambda^{(r)}}$

322 Accordingly, $\mathbf{H}_{f h}^{(r)}$ can be approximated by

$$
\mathbf{H}_{f h}^{(r)} \approx \frac{G\left(\hat{\mathbf{f}}^{(r)}+\mathbf{g}^{(r)} \delta\right)-G\left(\hat{\mathbf{f}}^{(r)}\right)}{\delta} \sqrt{\lambda^{(r)}}
$$

324 and the corresponding error is

$$
e r r=\sqrt{\lambda^{(r)}} G "\left(\hat{\mathbf{f}}^{(r)}\right) \frac{\left(\mathbf{g}^{(r)}\right)^{2} \delta}{2}+\ldots
$$

326 To evaluate $\mathbf{H}_{f h}^{(r)}$, we need to run the forward model $n_{s v d}$ (number of kept eigens) $\times$

$327 n_{\text {event }}$ (number of pumping or injection events) times.

328 If we further evaluate $G(\cdot)$ on $\hat{\mathbf{f}}^{(r)}-\mathbf{g}^{(r)} \delta$ and combine it with $G\left(\hat{\mathbf{f}}^{(r)}+\mathbf{g}^{(r)} \delta\right)$,

329 we have

330

$$
G\left(\hat{\mathbf{f}}^{(r)}+\mathbf{g}^{(r)} \delta\right)-G\left(\hat{\mathbf{f}}^{(r)}-\mathbf{g}^{(r)} \delta\right)=2\left[G^{\prime}\left(\hat{\mathbf{f}}^{(r)}\right) \mathbf{g}^{(r)} \delta+G^{\prime \prime \prime}\left(\hat{\mathbf{f}}^{(r)}\right) \frac{\left(\mathbf{g}^{(r)} \delta\right)^{3}}{3 !}+\ldots\right]
$$

331 Multiplying both sides with $\sqrt{\lambda^{(r)}}$ and $\mathbf{H}_{f h}^{(r)}$ can be approximated by

$$
\mathbf{H}_{f h}^{(r)} \approx \frac{G\left(\hat{\mathbf{f}}^{(r)}+\mathbf{g}^{(r)} \delta\right)-G\left(\hat{\mathbf{f}}^{(r)}-\mathbf{g}^{(r)} \delta\right)}{2 \delta} \sqrt{\lambda^{(r)}}
$$

333 The corresponding error is

$$
e r r=\sqrt{\lambda^{(r)}} G^{\prime \prime}\left(\hat{\mathbf{f}}^{(r)}\right) \frac{\left(\mathbf{g}^{(r)}\right)^{3} \delta^{2}}{3 !}+\ldots
$$

335 The evaluation of more accurate $\mathbf{H}_{f h}^{(r)}$ requires the exercise of forward model $2 n_{s v d} \times$ $n_{\text {event }}$ times. 


\subsection{Computational Advantages}

The proposed reduced-order dual state-parameter inverse algorithm is efficient when the number of kept leading eigens $\left(n_{s v d}\right)$ is less than 1500 . If the ratio of domain

341 size and correlation length is huge, large $n_{s v d}$ value increase the computational cost of $\operatorname{SVD}\left(O\left(n_{k l}^{3}\right)\right)$. Furthermore, evaluating $\mathbf{H}_{f h}^{(r)}$ through the forward or backward finite difference approach is efficient for many types of forward models (e.g., variable saturated diffusion equation, advection diffusion equation). It only requires executing the forward model for $n_{s v d} \times n_{\text {event }}$ (number of pumping events) times. On the contrary, when the forward model is elegant (e.g., fully saturated diffusion equation), it is cost-effective to evaluate the sensitivity of state with respect to unknown parameter (eq. 14, $\mathbf{J}_{f h}^{(r)}$ ) through the adjoint method. Only $n_{w}$ (number of observation wells) forward runs is required. In addition, updating state variable errors is efficient when $n_{d}$ (number of state variable) is less than 10000. The most expensive additional computational cost is to solve the inverse $n_{d} \times n_{d}$ matrix (eqs. 12 and 13) through either Cholesky or QR decompositions (matrix multiplication is an easy task under the 353 parallel computing scheme).

\section{3. Algorithm Verification}

356 In this section, three cases are used to examine the robustness of the proposed 357 algorithm. The first and second cases involve hydraulic tomographic surveys in a 358 synthetic aquifer without and with observation error, respectively. The third case is a 359 2-D application of tomography experiment in the field site. defined as 


$$
R^{2}=\left[\frac{\left(\mathbf{f}^{*}-\overline{\mathbf{f}}^{*}\right)^{T}\left(\hat{\mathbf{f}}^{(r)}-\overline{\mathbf{f}}^{(r)}\right)}{n_{f} \operatorname{std}\left(\mathbf{f}^{*}\right) \operatorname{std}\left(\hat{\mathbf{f}}^{(r)}\right)}\right]^{2}
$$

and

$$
L_{2}=\frac{\left(\mathbf{f}^{*}-\hat{\mathbf{f}}^{(0)}\right)^{T}\left(\mathbf{f}^{*}-\hat{\mathbf{f}}^{(0)}\right)}{n_{f}}
$$

365

366

367

368

369

370

371

372

373

374

375

376

377

378

are utilized to evaluate the similarity between the reference and estimated parameter fields. Overbar represents the average. $\operatorname{std}(\cdot)$ stands for the standard deviation.

\subsection{Observation-Error Free Synthetic Case}

The observation-error free synthetic case considers transient state HT in a two-dimensional horizontal confined aquifer of $30 \times 30$ square elements (figure 1). Each element is $1(\mathrm{~m}) \times 1(\mathrm{~m})$. The aquifer is bounded by the constant head boundary $(30 \mathrm{~m})$. The initial head is uniform $(30 \mathrm{~m})$ everywhere.

\section{(a) Forward Model}

The reference field (figure 1) is generated using a spectral method (Gutjahr, 1989; Robin et al., 1993) with mean geometric $T$ of one ( $\mathrm{m}^{2} /$ day), variance of $\ln T$ of one (-), and correlation scales of $10(\mathrm{~m})$ at both $\mathrm{x}$ and y directions. Eight wells (white dots) are evenly installed in the aquifer to collect the aquifer responses induced by three sequential pumping tests from early time till the system reaches steady state. The pumping wells are labeled with squares. The noise free observed heads only contain the numerical error (e.g., round-off and truncation errors), and its value is smaller than $10^{-7}(\mathrm{~m})$. The $S$ is a constant value of $0.001(-)$. The initial time step is 0.001 (day) and the maximum time step is 1 (day).

\section{(b) Inverse Model}

Assume $S$ is known and we would like to estimate the spatial $T$ distribution. The 
385 initial mean $T\left(\hat{\mathbf{f}}^{(0)}\right)$ is one $\left(\mathrm{m}^{2} /\right.$ day $)$, variance of $\ln T\left(\boldsymbol{\varepsilon}_{f f}^{(0)}\right)$ is one (-), variance of

386 observed head $\left(\boldsymbol{\varepsilon}_{h h}^{(0)}\right)$ is $10^{-4}\left(\mathrm{~m}^{2}\right)$, and the correlation lengths $\lambda_{x}$ and $\lambda_{y}$ are $10(\mathrm{~m})$.

\section{(c) Results of Estimate}

Figure 2 shows the performances of the estimated $T$ value using old algorithm (SLE) and figure 3 presents the performances using the new algorithm. Figure $2 \mathrm{a}$ presents the evolutions of mean squared error between the observed and simulated heads $\left(L_{2}\right.$ norm) and the spatial variance of $\ln T$ (Var $\left.\ln T\right)$ during the calibration process. Figure $2 \mathrm{~b}$ is the calibrated head at the final iteration. Figures $2 \mathrm{c}$ and $2 \mathrm{~d}$ are the final and best estimated $T$ field, respectively. Figure $2 \mathrm{e}$ and $\mathrm{f}$ are the scatter plots of the estimated $T$ verses reference $T$ corresponding to the final (figure $2 \mathrm{c}$ ) and best (figure 2d) estimated $T$ fields. As displayed in figure 2a, after $L_{2}$ norm approaches steady, the spatial variance of estimated $T$ (pink line) still increases with a constant rate. The gaining of spatial variation of estimated $T$ values comes from the over calibrated observed head. Due to the natural of least squared algorithm (e.g., minimizing the mean squared error of state variables), the algorithm compensates the numeric errors by adjusting the estimated $T$ to unreasonably high and low values, although the general spatial trend of the estimated $T$ fields remains similar. As the result, compare to the best estimate of $T$ field (figure $2 \mathrm{~d}$ and f), the final estimate diverges (figure $2 \mathrm{c}$ and $\mathrm{e}$ ).

On the contrary, estimate using the new algorithm does not encounter the divergence issue. As shown in the calibration process (figure 3a), the spatial variance of estimated $T$ (pink line) reaches steady after $L_{2}$ decays to the value of $10^{-10}$ (i.e., magnitude of numeric error). The final estimated field (figure 3c) converges and the performances in terms of the statistical indices (figure 3d), namely $L_{2}, R^{2}$ (determination coefficient), or the slope and intercept of the fitted linear relationship 
410 between the estimates and the true values, are equally good as the best estimate by the

411 previous algorithm (figure 2c). In other word, the new algorithm eliminates the over

412 fitting issue.

\section{3.2. Noisy Synthetic Case}

415 This example aims to reveal the advantages of the algorithm when the 416 measurement errors are presented. To accomplish this goal, the Gaussian noises with 417 standard deviation of $10^{-3}(\mathrm{~m})$ are superimposed on the observed heads discussed in 418 section 3.1. The design of inverse model is identical with those explained in section $419 \quad 3.1$

Figure 4 shows the performances (evolution of calibration process, head fitting, 421 contour of the estimate field, and the scatter plot between the estimate and reference fields) of the estimated $T$ value using original algorithm, and figure 5 presents the

423 performances using the new one. By comparing the final estimate with the manually

424 selected best estimate of original SLE (figure $4 \mathrm{~d}$ and $\mathrm{f}$ ), the final estimated $T$ field

425 diverges as indicated by the increase in variance of $\ln T$ (pink line in figure $4 a$ ), unreasonable high and low values (red and blue spots in figure 4c) of the final

427 estimated $T$ fields, and the uncorrelated estimate and reference $\ln T$ values (figure $4 \mathrm{e}$ ).

428 On the contrary, the final estimate using the proposed algorithm shows that the 429 estimated field converges to the reasonable spatial pattern and values. The variance of $430 \ln T$ (pink line in figure 5a) approaches stable and the simulated heads reproduce the 431 adjusted observed heads (sum of observed heads and estimated head errors, figure 5b).

432 Furthermore, the contour map and scatterplot of the final estimate (figure $5 \mathrm{c}$ and $\mathrm{d}$ )

433 suggest the estimated field is close to the manually selected best estimate of original 434 SLE (figure $4 \mathrm{~d}$ and $\mathrm{f}$ ) and the reference (figure 1). This means the new method no 435 longer overestimate the parameter fields and can automatically converge to an optimal 
436 estimate under the given constrains.

437

438

\subsection{Field Data}

The proposed algorithm is applied to a river stage tomographic survey conducted in Pingtun Plain, Taiwan. It is a $1200 \mathrm{~km}^{2}$ catchment with three major rivers penetrating from the north to south (figure 6). The plain is bound by foothills and river valleys at the north, faults at the west and east, and the shoreline at the south. As illustrated in figure $6 \mathrm{~b}$, the geology inferred from well logs shows that the upstream subsurface is consist of gravel. Follows by the layered sand and clay structure at middle and down streams. The regions with unconsolidated coarse sediments (gravel and sand) are aquifer and with fine sediments (silt and clay) are aquitard. The aquitard is characterized as marine deposition because abundant fossils such as shells and foraminifera live in the shallow marine and lagoon are discovered. The aquifer is characterized as non-marine deposition. Figure $6 \mathrm{c}$ presents the stream stage and groundwater level variations during 2006. The average annual rainfall is $2500 \mathrm{~mm}$, with most of the precipitation happen between May and September.

We focus on characterizing the heterogeneity of shallow aquifer because it is the major water source of agriculture, industrial, and municipal water supply. The average aquifer thickness is $40 \mathrm{~m}$. This catchment is discretized into a two-dimensional horizontal confined aquifer with 5619 elements. Each element is $0.5(\mathrm{~km}) \times 0.5(\mathrm{~km})$. There are 36 monitoring wells evenly placed across the catchment and measuring the hourly groundwater level variation of the aquifer since 1998. The aquifer is bounded by the time varying head boundary. The time varying heads along the boundary are extrapolated by kriging using the observed head collected from all of the monitoring wells. Water levels collected from stream gauges are incorporated into the diffusion wave equation to estimate the stream stages along the river. These estimated stages 
462 are then treated as the prescribed head in the groundwater model. The initial 463 groundwater level is estimated by spinning up the model for 6 years prior to June 4642006 utilizing the effective $T\left(1\left(\mathrm{~m}^{2} /\right.\right.$ day) $)$, effective $S\left(10^{-5}(-)\right)$, time varying head

465 boundary, and stream stage variations.

The denoised groundwater levels from June to September 2006 are selected using the strategy (i.e., wavelet) discussed in Wang et al. (2017). There is a total of 1440 measured heads selected for river stage tomographic survey. The initial mean $T$ $\left(\hat{\mathbf{f}}^{(0)}\right)$ is $1\left(\mathrm{~m}^{2} /\right.$ day $)$, variance of $\ln T\left(\boldsymbol{\varepsilon}_{f f}^{(0)}\right)$ is one $(-)$, variance of observed head $\left(\boldsymbol{\varepsilon}_{h h}^{(0)}\right)$ is $10^{-4}\left(\mathrm{~m}^{2}\right)$, and the correlation lengths $\lambda_{x}$ and $\lambda_{y}$ are $15(\mathrm{~km})$. For simplicity, we assume $S$ is uniform and focus on estimating the spatial $T$ distribution. The patterns of estimated $T$ fields should be consistent with the hydraulic diffusivity field.

Figure 7 presents the calibration using the original SLE algorithm. The increasing of variance of $\ln T$ (figure 7a) near the end of iteration (iteration 100) suggests the estimate diverges, although the parameter field reproduces the observed drawdowns (figure 7b). The unreasonable huge spatial $T$ variation corresponds to the significantly low and high values on the contour map of the final estimate field (figure 7c). The contour map of manually selected best estimate is shown in figure $7 \mathrm{~d}$ and the calibrated heads are similar with those in figure $7 \mathrm{~b}$.

Figure 8 shows the estimate using the new algorithm. The performance clearly demonstrates the robustness and usefulness of the new algorithm on characterizing the subsurface heterogeneity. Compared with the variance of $\ln T$ in figure $7 \mathrm{a}$, it stabilizes at the end of iteration (figure 8a) while still reproduces the adjusted observed heads (figure $8 \mathrm{~b}$ ). The estimated field (figure 8c) shares the similar spatial patterns with the manually selected one (figure 7d).

To further examine the reliability of the estimate, the estimated $T$ field is 
487 compared with the map of geological sensitivity regions (figure 9) delineated by the represent the major areas water recharges the aquifer. They are categorized by core samples, geophysics (e.g., electric resistivity), and geochemical survey. In general, the

491 deposition of geological sensitivity region is gravel and the aquifer thickness is greater than $100 \mathrm{~m}$ (blue areas in figure 6b). Compared figure 9 with figure $7 \mathrm{~d}$ and $8 \mathrm{c}$,

493 the high $T$ regions located near the upper streams (red areas) are in parallel with the

494 geological sensitivity regions.

495

496

497

498

\section{Conclusion}

In this paper, a reduced order geostatistical model is developed to account for the subsurface heterogeneity. This method includes the evaluation of the errors of state variables and unknown parameters to improve the robustness of convergence. The over fitting problem (i.e., diverged estimated parameter fields) is leveraged by considering these errors into the calibration process. The memory burden (i.e. high dimensional parameter covariance) and requirement of domain shape (e.g., brick or rectangle) are also relieved by approximating the parameter covariance matrix through limited number of leading eigenvalues and eigenvectors using SVD. Meanwhile, the computation of sensitivity is replaced by the direct evaluation of cross-covariance through the finite differencing method. The modification relaxes barrier of implementing this inverse algorithm to different disciplines because the derivation of adjoint state method is no longer necessary. Lastly, as the stability of convergence is robust and the evaluation of cross-covariance (sensitivity) is efficient, the proposed algorithm is valuable and attractive for multi-discipline scientific problems, especially useful and convenient for assimilating different types of measurements. 


\section{5. Acknowledgment}

515 This research is in part by U.S. Civilian Research and Development Foundation

516 (CRDF Global) under the award number (DAA2-15-61224-1): Hydraulic tomography

517 in shallow alluvial sediments: Nile River Valley, Egypt. The authors gratefully

518 acknowledge the financial support by Minster of Science and Technology, Taiwan

519 under award number 108-2116-M-002-029-MY3.

520

\section{Reference}

522 Ait-El-Fquih, B., El Gharamti, M., and Hoteit, I.: A Bayesian consistent dual

523 ensemble Kalman filter for state-parameter estimation in subsurface hydrology,

524 Hydrol. Earth Syst. Sci., 20, 3289-3307, https://doi.org/10.5194/hess-20-3289-2016, 5252016.

526

Cirpka, O. A., and P. K. Kitanidis: Sensitivity of temporal moments calculated by the

528 adjoint-state method and joint inversing of head and tracer data, Adv. Water Resour., 24(1), 89-103, doi:10.1016/S0309-1708(00)00007-5, 2000.

530

531 Field G, Tavrisov G, Brown C, Harris A, Kreidl OP: Particle filters to estimate 532 properties of confined aquifers. Water Resour. Manag., 30(9):3175-3189,

533 https://doi.org/10.1007/s11269-016-1339-1, 2016.

535 Ghanem, R. G., \& Spanos, P. D.: Stochastic finite elements: A spectral approach. 536 North Chelmsford, MA: Courier Corporation, 2003. 
539 assimilation for large-scale systems: the spectral Kalman filter. Adv. Water Resour.,

540 86, 260-272, doi:10.1016/j.advwatres.2015.07.017, 2015.

541

542 Gutjahr, A. L.: Fast Fourier transforms for random field generation, project report for

543 Los Alamos grant, Contract 4-R58-2690R, Dep. of Math., N. M. Tech., Socorro, 5441989.

545

546 Horning, S., J. Sreekanth, A. Bardossy: Computational efficient inverse groundwater

547 modeling using Random Mixing and Whittaker-Shannon interpolation, Adv. Water

548 Resour., 123, 109-119, doi:10.1016/j.advwatres.2018.11.012, 2019.

549

550 Illman, W. A., S. J. Berg, and Z. Zhao: Should hydraulic tomography data be

551 interpreted using geostatistical inverse modeling? A laboratory sandbox investigation,

552 Water Resour. Res., 51, 3219-3237, doi:10.1002/2014WR016552, 2015.

553

554 Kitanidis, P. K.: Quasi-linear geostatistical theory for inversing, Water Resour. Res.,

555 31(10), 2411-2419, doi:10.1029/95WR01945, 1995.

556

557 Kitanidis, P. K., and Lee, J.: Principal Component Geostatistical Approach for large-

558 dimensional inverse problems, Water Resour. Res., 50, 5428-5443,

559 doi:10.1002/2013WR014630, 2014.

560

561 Kitanidis PK. Compressed state Kalman filter for large systems, Adv. Water Resour.,

76, 120-126, 2015, doi:10.1016/j.advwatres.2014.12.010.

564 Leng, C. H., and Yeh, H. D.: Aquifer parameter identification using the extended 
565 Kalman filter, Water Resour. Res., 39, 1062, doi:10.1029/2001WR000840, 3, 2003.

566

567 Li, J. Y., Ambikasaran, S., Darve, E. F., and Kitanidis, P. K.: A Kalman filter powered

568 by $H^{2}$-matrices for quasi-continuous data assimilation problems, Water Resour.

569 Res., 50, 3734-3749, doi:10.1002/2013WR014607, 2014.

570

571 Li, J. Y., Kokkinaki, A., Ghorbanidehno, H., Darve, E. F., and Kitanidis, P. K.: The

572 compressed state Kalman filter for nonlinear state estimation: Application to large-

573 scale reservoir monitoring, Water Resour. Res., 51, 9942-9963,

574 doi:10.1002/2015WR017203, 2015.

575

576 Lin, Y., O'Malley, D., and Vesselinov, V. V.: A computationally efficient parallel

577 Levenberg - Marquardt algorithm for highly parameterized inverse model

578 analyses, Water Resour. Res., 52, 6948-6977, doi:10.1002/2016WR019028, 2016.

579

580 Lin, Y., Le, E. B., O'Malley, D., Vesselinov, V. V., and Bui-Thanh, T.: Large-scale

581 inverse model analyses employing fast randomized data reduction, Water Resour.

582 Res., 53, 6784- 6801, doi:10.1002/2016WR020299, 2017.

583

584 Liu, X., Zhou, Q., Birkholzer, J., and Illman, W. A.: Geostatistical reduced-order

585 models in underdetermined inverse problems, Water Resour. Res., 49, 6587- 6600,

586 doi:10.1002/wrcr.20489, 2013.

587

588 Liu, X., Zhou, Q., Kitanidis, P. K., and Birkholzer, J. T.: Fast iterative implementation

589 of large-scale nonlinear geostatistical inverse modeling, Water Resour. Res., 50, 198-

590 207, doi:10.1002/2012WR013241, 2014. 
592 Nowak, W., and O. A. Cirpka: Geostatistical inference of hydraulic conductivity and

593 dispersivities from hydraulic heads and tracer data, Water Resour. Res., 42, W08416, 594 doi:10.1029/2005WR004832, 2006.

595

596 Nowak, W., Litvinenko, A.: Kriging and spatial design accelerated by orders of 597 magnitude: combining low-rank covariance approximations with fft-techniques. Math. 598 Geosci. 45 (4), 411-435, doi:10.1007/s11004-013-9453-6, 2013.

599

600 Pagh, R.: Compressed matrix multiplication. ACM Trans. Comput. Theory, 601 5(3):9:1-9:17, doi: 10.1145/2493252.2493254, 2013.

602

603 Robin, M. J. L., A. L. Gutjahr, E. A. Sudicky, and J. L. Wilson: Cross-correlated 604 random field generation with the direct Fourier transform method, Water Resour. Res., 605 29, 2385-2397, doi:10.1029/93WR00386, 1993.

606

607 Saibaba, A. K., and Kitanidis, P. K.: Efficient methods for large-scale linear inversion 608 using a geostatistical approach, Water Resour. Res., 48, W05522, 609 doi:10.1029/2011WR011778, 2012.

610

611 Schöniger, A., Nowak, W., and Hendricks Franssen, H.-J.: Parameter estimation by 612 ensemble Kalman filters with transformed data: Approach and application to 613 hydraulic tomography, Water Resour. Res., 48, W04502, doi:10.1029/2011WR010462, 6142012. 
617 Sensitivity analysis and parameter identification, Water Resour.

618 Res., 26(10), 2507-2525, doi:10.1029/WR026i010p02507, 1990.

619

620 Sykes, J. F., J. L. Wilson, and R. W. Andrews: Sensitivity Analysis for Steady-State

621 Groundwater Flow Using Adjoint Operators, Water Resour. Res., 21(3), 359-371,

622 doi:10.1029/WR021i003p00359, 1985.

623

624 Tsai, J.-P., Yeh, T.-C. J., Cheng, C.-C., Zha, Y., Chang, L.-C., Hwang, C., Wang, Y.-L.,

625 Hao, Y.: Fusion of time-lapse gravity survey and hydraulic tomography for estimating 626 spatially varying hydraulic conductivity and specific yield fields. Water Resour. Res., 627 53, 8554-8571. doi:10.1002/2017WR020459, 2017.

628

629 Vesselinov, V., S. Neuman, and W. Illman: Three-dimensional numerical inversion of 630 pneumatic cross-hole tests in unsaturated fractured tuff 1. Methodology and borehole 631 effects, Water Resour. Res., 37, 3001-3017, doi:10.1029/2000WR000133, 2001.

632

633 Yeh, T.-C. J., M. Jin, and S. Hanna: An Iterative Stochastic Inverse Method: 634 Conditional Effective Transmissivity and Hydraulic Head Fields, Water Resour. Res., 635 32(1), 85-92, doi:10.1029/95WR02869, 1996.

636

637 Yeh, H. D., and Y. C. Huang: Parameter estimation for leaky aquifers using the 638 639 Hydrol., 302 (1-4), 28-45, doi:10.1016/j.jhydrol.2004.06.035, 2005.

640

641 Yin, D., and W. A. Illman: Hydraulic tomography using temporal moments of 642 drawdown recovery data: A laboratory sandbox study, Water Resour. Res., 45, 
645 Zha, Y., T.-C. J. Yeh, W. A. Illman, W. Zeng, Y. Zhang, F. Sun, L. Shi: A

646 reduced-order successive linear estimator for geostatistical inversion and its

647 application in hydraulic tomography. Water Resour. Res., 54, 1616-1632,

648 doi:10.1002/2017WR021884, 2018.

649

650 Zhang, D. and Lu, Z.: An efficient, high-order perturbation approach for flow in

651 random porous media via Karhunen-Loève and polynomial expansions. Journal of

652 Computational Physics, 194 (2), 773- 794. https://doi.org/10.1016/j.jcp.2003.09.015, 6532004.

654

655 Zhang, H., Hendricks Franssen, H.-J., Han, X., Vrugt, J. A., and Vereecken, H.: State

656 and parameter estimation of two land surface models using the ensemble Kalman

657 filter and the particle filter, Hydrol. Earth Syst. Sci., 21, 4927-4958,

658 https://doi.org/10.5194/hess-21-4927-2017, 2017.

659

660 Zhang, J., Lin, G., Li, W., Wu, L., Zeng, L.: An iterative local updating ensemble

661 smoother for estimation and uncertainty assessment of hydrologic model parameters

662 with multimodal distributions. Water Resour. Res., 54, 1716-1733,

663 doi:10.1002/2017WR020906, 2018.

664

665 Zunino A. and Mosegaard K.: An efficient method to solve large linearizable inverse

666 problems under Gaussian and separability assumptions. Computers and

667 Geosciences, 122, 77-86, doi:10.1016/j.cageo.2018.09.005, 2019. 


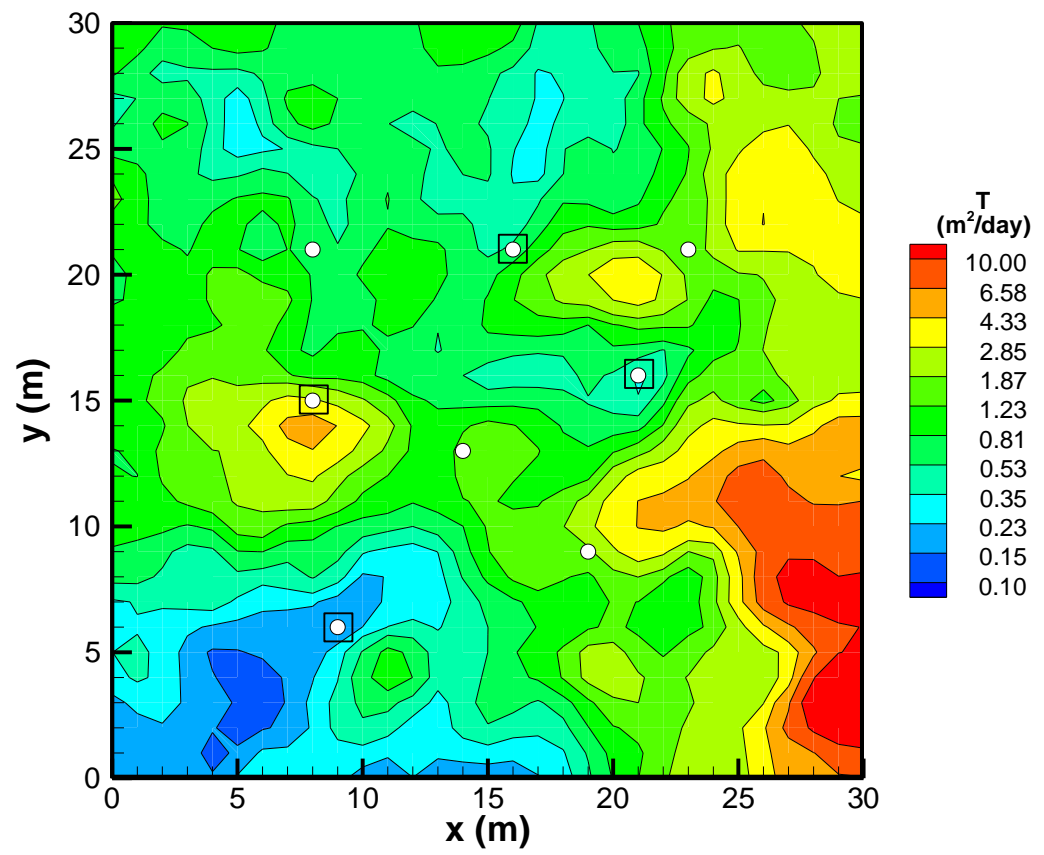

669

670 Figure 1. Reference hydraulic transimissivity $T\left(\mathrm{~m}^{2} /\right.$ day) field. The white dots 671 represent monitoring wells and the squared are pumping wells. Four boundaries are 672 the constant head.

673 
674

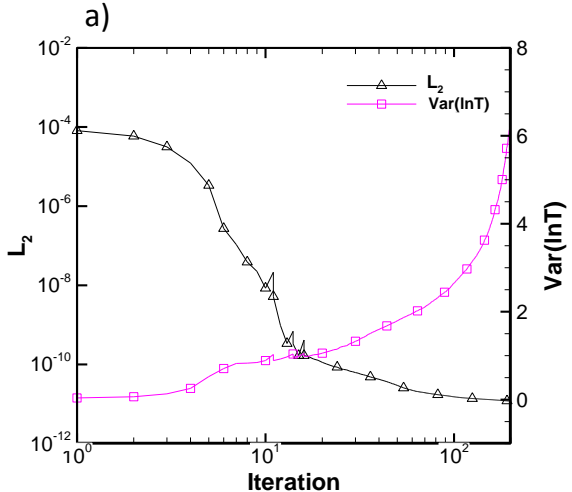

c)

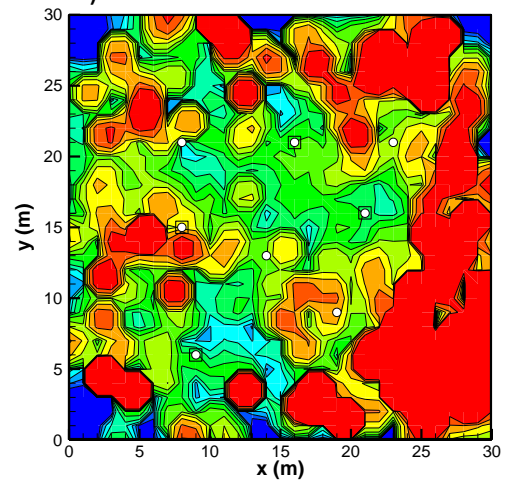

675

676

677

678

679

680

681

682

683

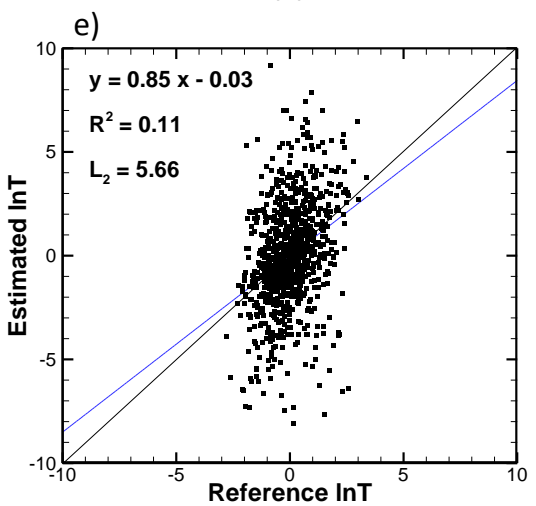
$\ln T$ b)

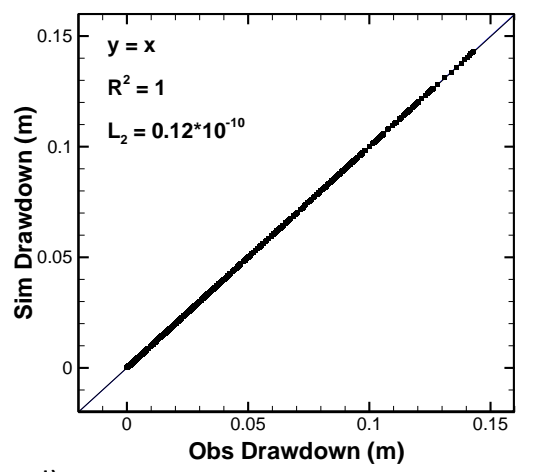

d)
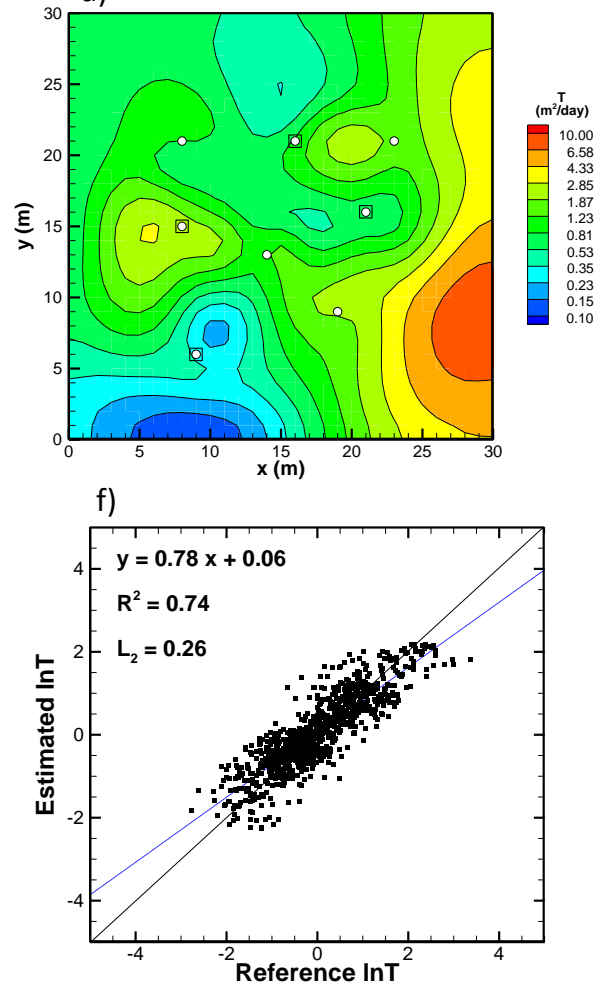

Figure 2. The estimated hydraulic transmissivity $T\left(\mathrm{~m}^{2} /\right.$ day) field using noise free observed head and old algorithm. a) The evolutions of mean squared error between the observed and simulated heads $\left(L_{2}\right.$ norm) and the spatial variance of $\ln T(\operatorname{Var} \ln T)$ during the calibration process. b) The calibrated head of the final iteration. c) The final estimated $T$ field. d) The best estimated $T$ field. e) The scatter plots of final estimated verses reference $\ln T$. f) The scatter plots of best estimated verses reference 
685

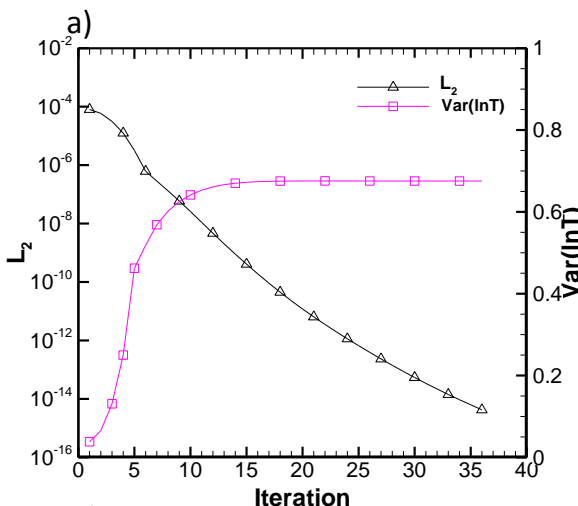

c)

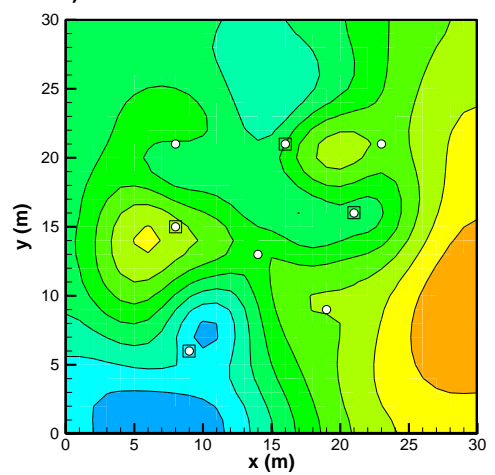

b)

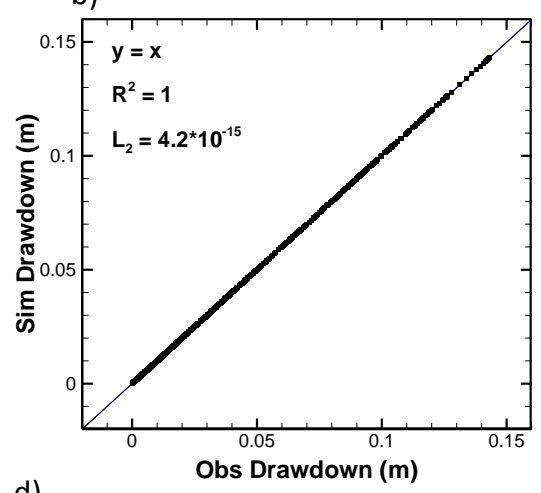

d)

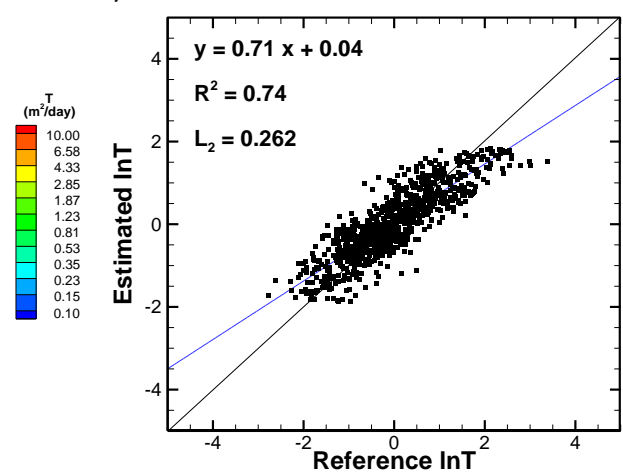

686

687

688

689

690

691

692

693

Figure 3. The estimated hydraulic transmissivity $T\left(\mathrm{~m}^{2} /\right.$ day) field using noise free observed head and new algorithm. a) The evolutions of mean squared error between the observed and simulated heads ( $L_{2}$ norm) and the spatial variance of $\ln T$ (Var $\left.\ln T\right)$ during the calibration process. b) The calibrated head of the final iteration. c) The final estimated $T$ field. d) The scatter plots of final estimated verses reference $\ln T$. 
694

695

696
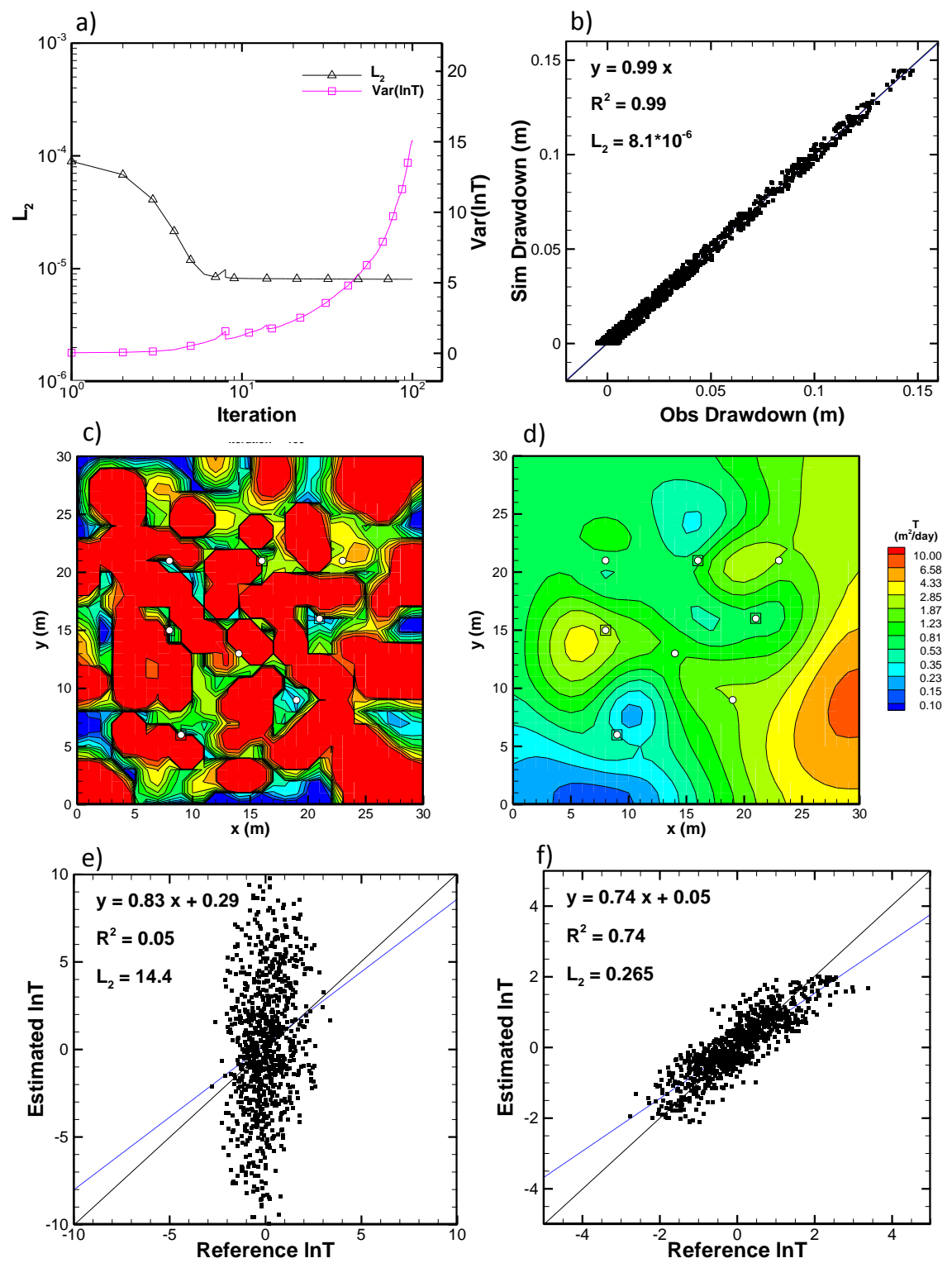

Figure 4. The estimated hydraulic transmissivity $T\left(\mathrm{~m}^{2} /\right.$ day) field using noisy observed head and old algorithm. a) The evolutions of mean squared error between the observed and simulated heads $\left(L_{2}\right.$ norm) and the spatial variance of $\ln T$ (Var $\left.\ln T\right)$ during the calibration process. b) The calibrated head of the final iteration. c) The final estimated $T$ field. d) The best estimated $T$ field. e) The scatter plots of final estimated verses reference $\ln T$. f) The scatter plots of best estimated verses reference $\ln T$. 

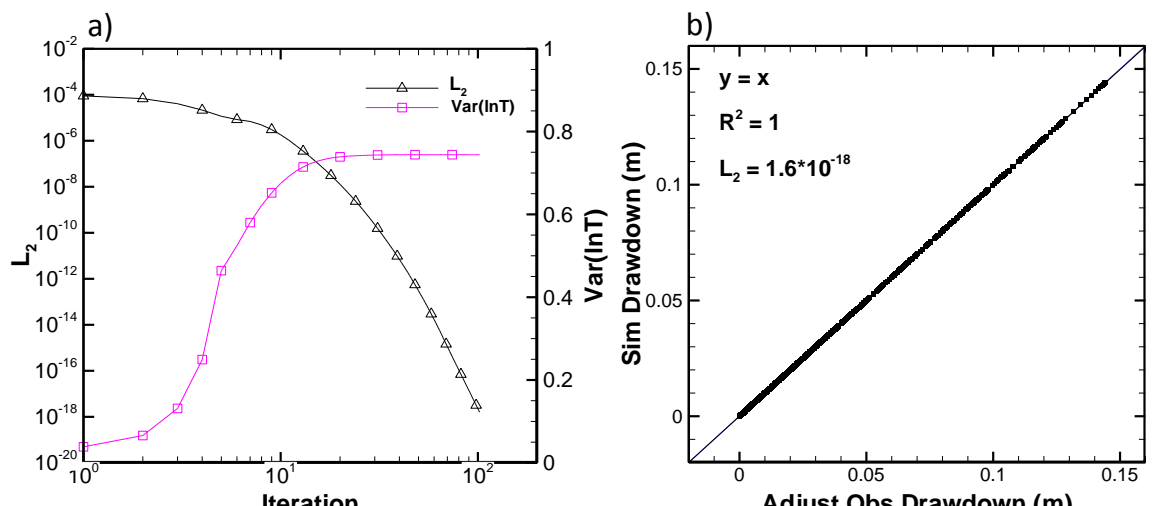

c)

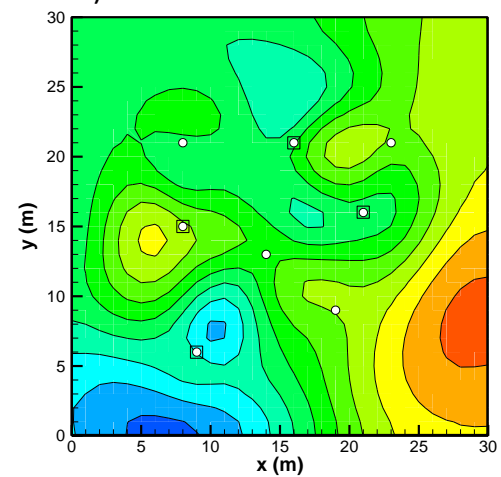

d)

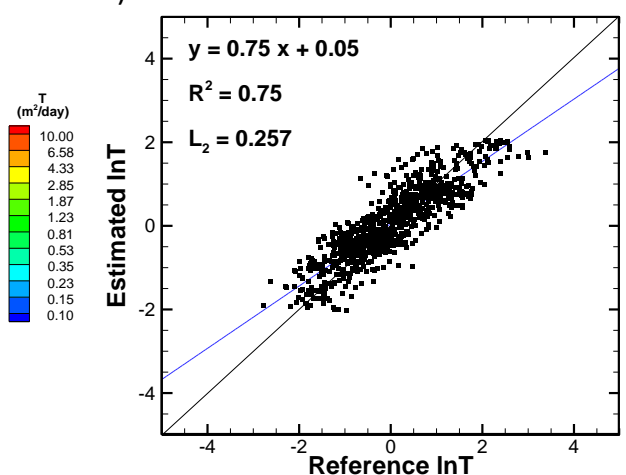

706

707

708

709

710

711

712

713

Figure 5. The estimated hydraulic transmissivity $T\left(\mathrm{~m}^{2} /\right.$ day) field using noisy observed head and new algorithm. a) The evolutions of mean squared error between the observed and simulated heads ( $L_{2}$ norm) and the spatial variance of $\ln T$ (Var $\ln T$ ) during the calibration process. b) The calibrated heads verse adjusted observed heads (observed head + estimated error) of the final iteration. c) The final estimated $T$ field. d) The scatter plots of final estimated verses reference $\ln T$. 


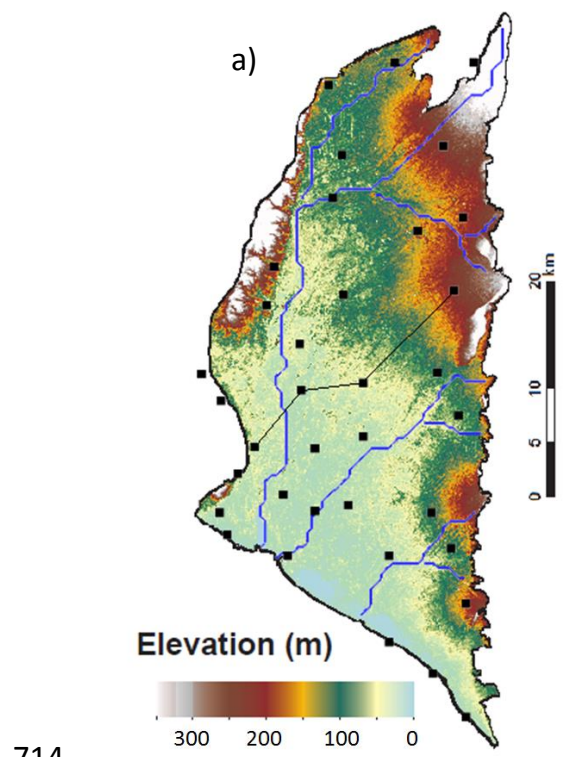

714

b) $\square$ Gravel

50

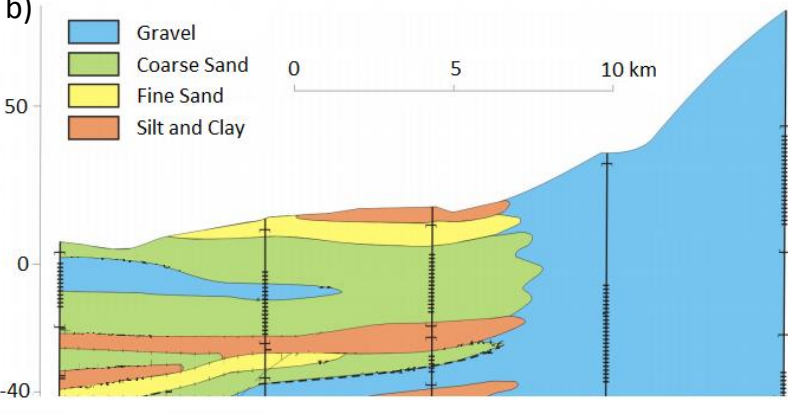

$$
0
$$

715

c)

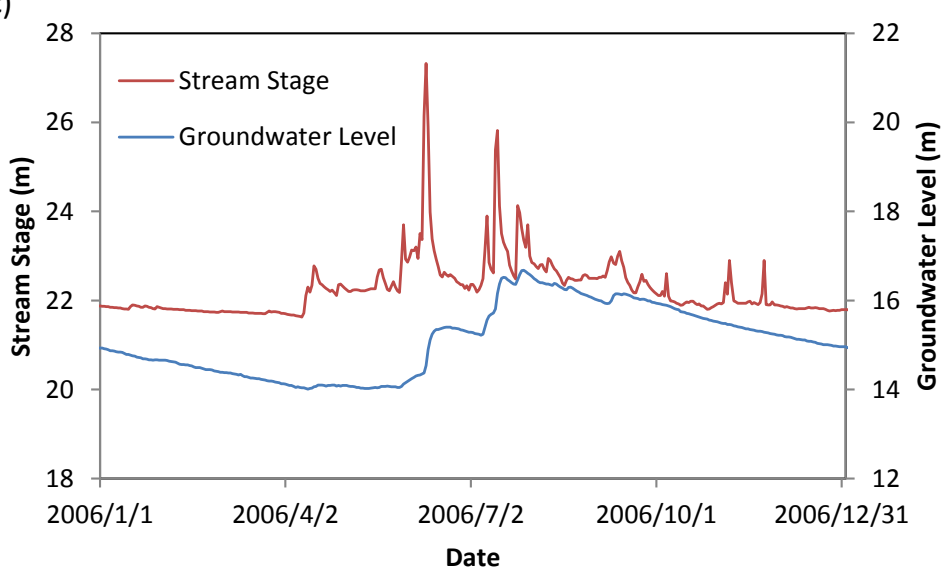


https://doi.org/10.5194/hess-2019-622

Preprint. Discussion started: 3 January 2020

(C) Author(s) 2020. CC BY 4.0 License.

717 Figure 6. a) Topography of the study plain. The blue lines represent rivers and the

718 black rectangles are groundwater monitoring wells. The black line is geological cross

719 section. b) Geological cross section. c) Stream stage and groundwater level variations

720 during 2006. a) and b) are modified from the website of Water Resources Agency, the

721 administrative agency of the Ministry of Economic Affairs in Taiwan.

722

723

724

725 
726

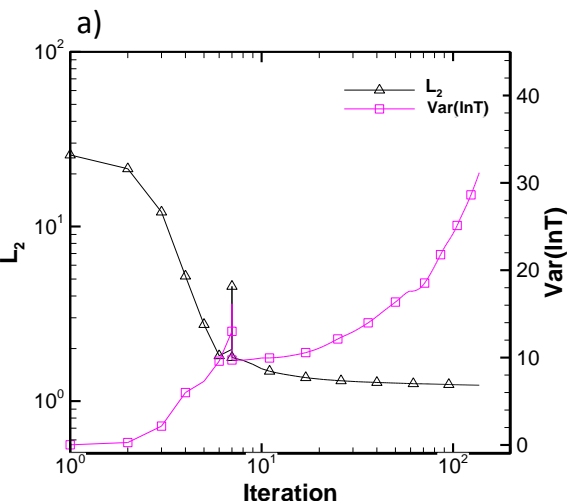

c)

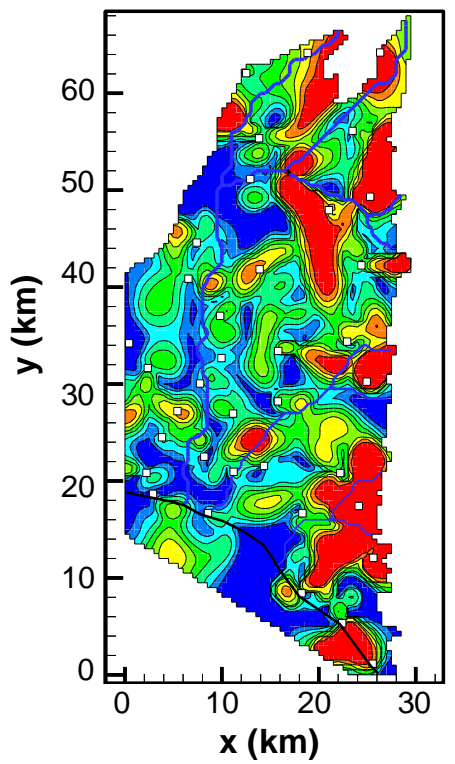

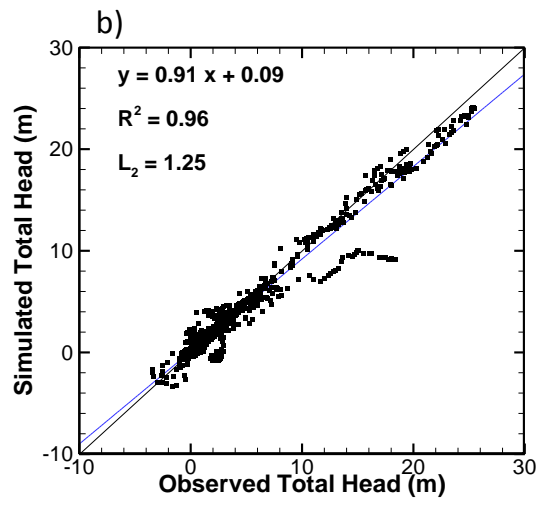

d)

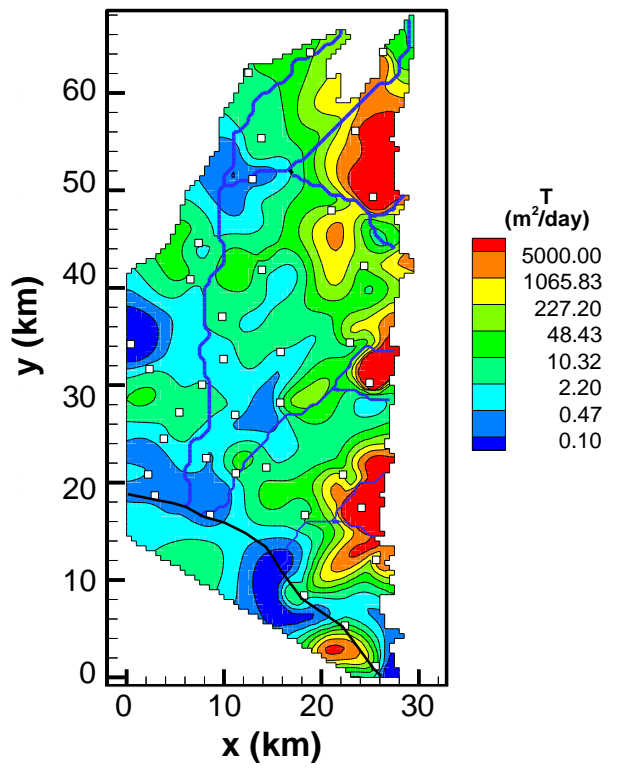

Figure 7. The estimated hydraulic transmissivity $T\left(\mathrm{~m}^{2} /\right.$ day) field using observed head in the field and old algorithm. a) The evolutions of mean squared error between the observed and simulated heads ( $L_{2}$ norm) and the spatial variance of $\ln T$ (Var $\ln T$ ) during the calibration process. b) The calibrated head of the final iteration. c) The final estimated $T$ field. d) The best estimated $T$ field. The white squares represent wells, the blue lines are rivers, and the black line is shoreline. 

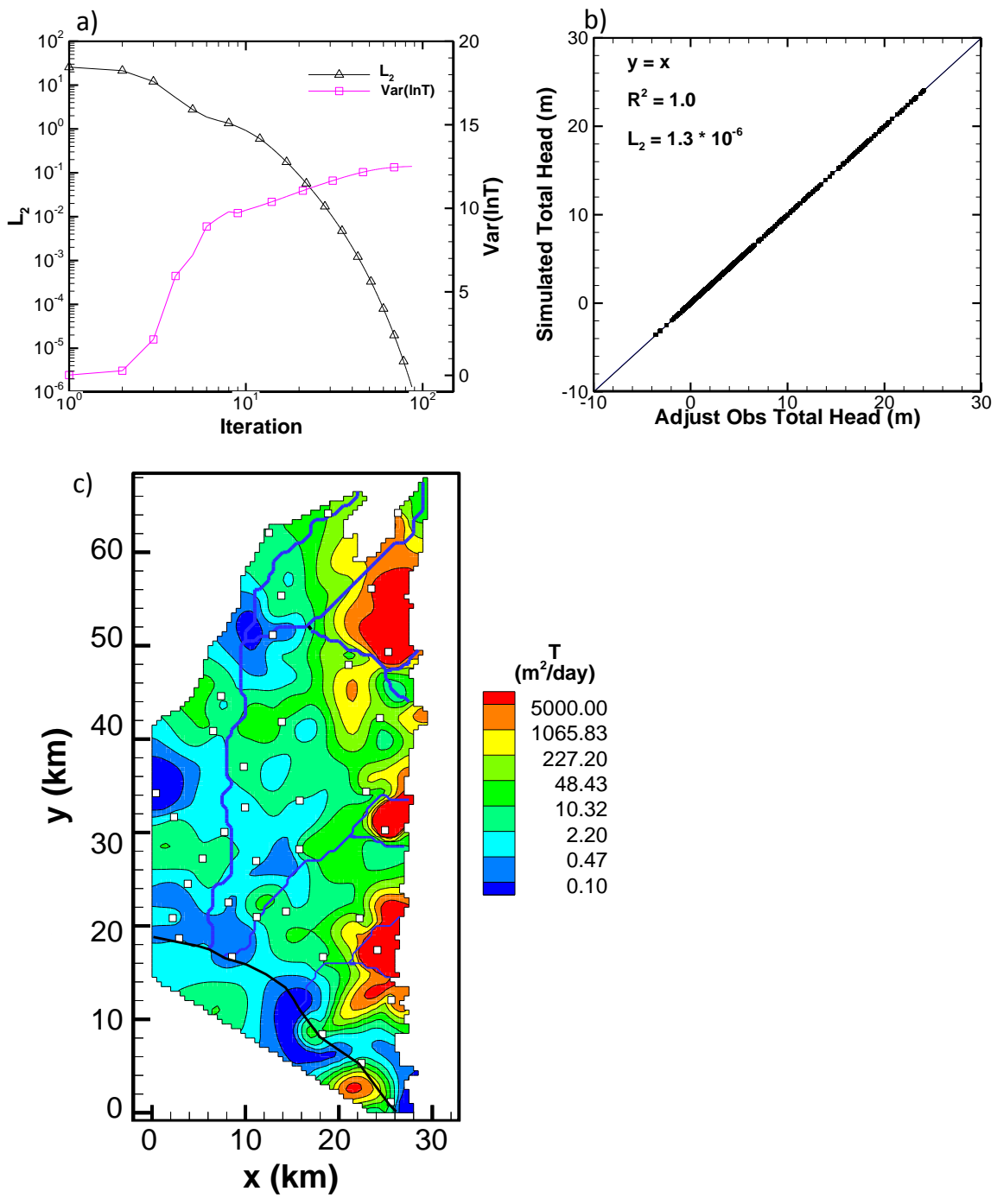

738 Figure 8 . The estimated hydraulic transmissivity $T\left(\mathrm{~m}^{2} /\right.$ day) field using observed head

739 in the field and new algorithm. a) The evolutions of mean squared error between the

740 observed and simulated heads $\left(L_{2}\right.$ norm) and the spatial variance of $\ln T(\operatorname{Var} \ln T)$

741 during the calibration process. b) The calibrated heads verse adjusted observed heads

742 (observed head + estimated error) of the final iteration. c) The final estimated $T$ field.

743 The white squares represent wells, the blue lines are rivers, and the black line is

744 shoreline. 


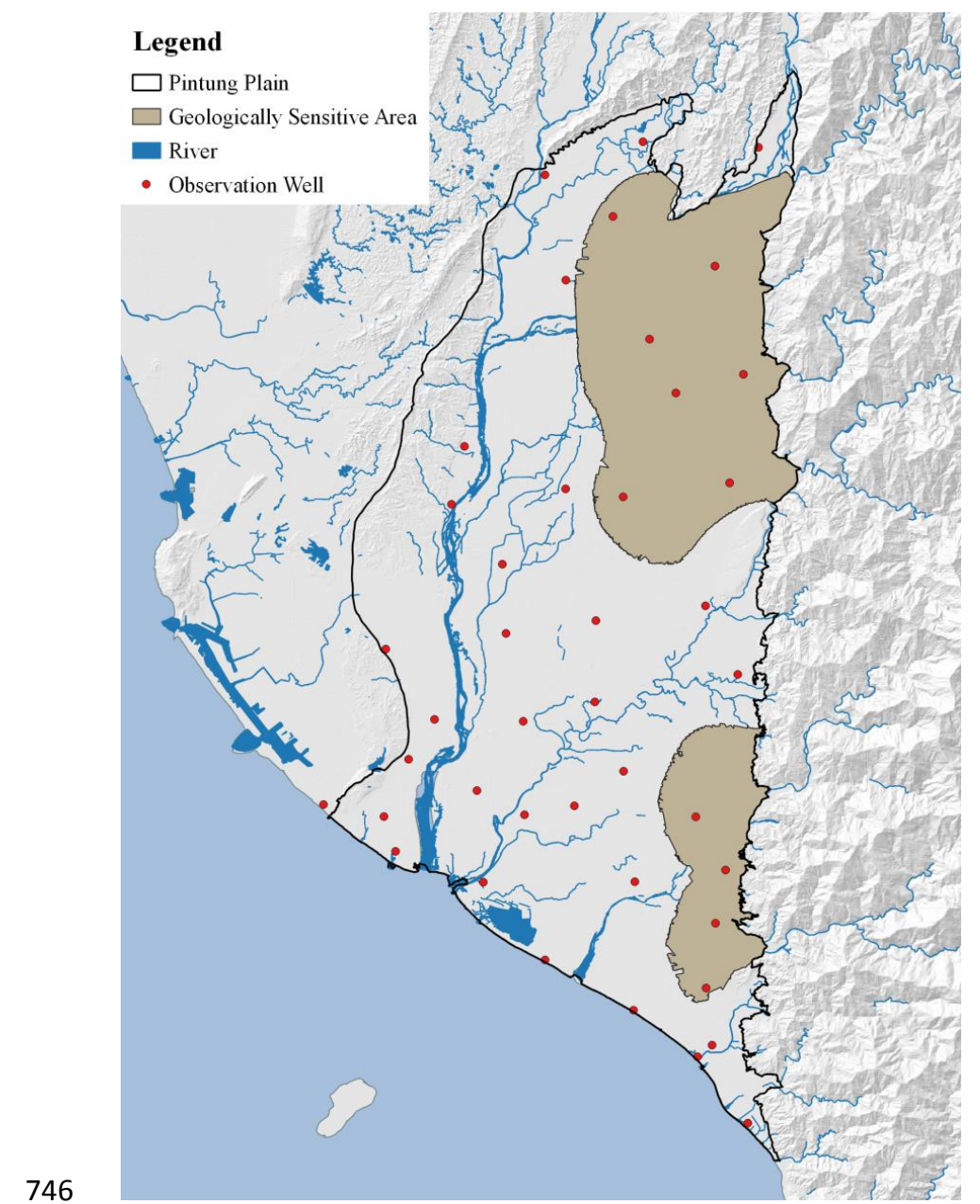


https://doi.org/10.5194/hess-2019-622

Preprint. Discussion started: 3 January 2020

(c) Author(s) 2020. CC BY 4.0 License.

\section{Code/Data availability}

751 The code and data are available upon the request through corresponding author.

752

753 Author contribution

754 Y.-L. Wang designed the study, carried out the analysis, interpreted the data, and

755 wrote the paper. T.-C. Jim Yeh and J.-P. Tsai provided the financial support and helped

756 finalize the paper.

757

758 Competing interests

759 The authors declare that they have no conflict of interest.

760 